\title{
Solar geoeffective phenomena: Impact on the near-Earth space and the opportunity to forecast
}

\author{
V. N. Ishkov ${ }^{1}$ \\ Received 22 November 2007; accepted 7 April 2008; published 23 April 2008.
}

[1] The solar research of the last decade has left no doubt that geoeffective phenomena on the Sun, which dynamically affect the state of near-Earth space (NES) are extremely large flare events and coronal holes. We attribute to the class of flare phenomena solar flares with a complete spectrum of dynamic manifestations of the mass motion and radiation in all ranges of the electromagnetic spectrum as well as solar filament eruptions with all accompanying phenomena. The possibility to predict geoeffective solar phenomena and their effects on NES is based on the analysis of correlations: emergence of new magnetic fluxes in the solar atmosphere, their physical characteristics, and the character of their interaction with the already present magnetic field; time distribution of large flares within active regions; temporal and spatial distribution of solar filament eruptions; formation, distribution, and evolution of solar coronal holes $(\mathrm{CH})$. The agents causing NES disturbances are transient structures: coronal mass ejections, which result from active processes in flares and filament eruptions; high-speed flows of solar plasma following a shock wave from large solar flares and solar filament eruptions or outflowing from regions with an open magnetic configuration. Prediction for the beginning of NES disturbances and of their duration is possible for a period from 1 to 5 days and for coronal holes for a period of one solar revolution (27.3 days). This makes possible planning various technological, biological, and medical experiments together with protective measures for the team and instruments of space laboratories. INDEX TERMS: 7536 Solar Physics, Astrophysics, and Astronomy: Solar activity cycle; 7899 Space Plasma Physics: General or miscellaneous; 7974 Space Weather: Solar effects; 7984 Space Weather: Space radiation environment; KEYWORDS: Solar geoeffective phenomena, near-Earth space, forecasting, cyclicity of solar activity.

Citation: Ishkov, V. N. (2008), Solar geoeffective phenomena: Impact on the near-Earth space and the opportunity to forecast, Russ. J. Earth. Sci., 10, ES3005, doi:10.2205/2007ES000247.

\section{Introduction}

[2] The beginning of the space era became a new step in the study of the Earth in a broad sense, from its interiors up to the interface with the heliosphere, by ground-based as well as spaceborne instruments. The integration of observations from the Earth and space within the framework of the International Geophysical Year (1957-1958) gave a stimulus to all science such that its development follows just the path of comparison and consideration of measurements from space and from the ground. The ever increasing rate of space

\footnotetext{
${ }^{1}$ Institute of Terrestrial Magnetism, Ionosphere, and Radiowave Propagation, Russian Academy of Sciences, Troitsk, Moscow Region, Russia
}

Copyright 2008 by the Russian Journal of Earth Sciences. ISSN: 1681-1208 (online) exploration has set a problem of assessment and prediction of the NES environment state and in any given region of the heliosphere.

[3] In this paper we mean for near-Earth space the region beginning from heights $50-60 \mathrm{~km}$ above the Earth and extending to distances of tens of Earth radii up to the interface with the heliosphere; in the heliosphere, the material that fills it is still connected to our planet, not with the Sun or any other celestial body. It is clear that in this region we deal mainly with natural plasma.

[4] For "space weather" we mean the state of the upper Earth's atmosphere (mesosphere, thermosphere), magnetosphere, ionosphere, all layers of near-Earth space in any given time interval. By present it has been understood that the main effect on near-Earth space is exerted by the Sun and by geoeffective phenomena in its atmosphere. However, the events of the last decade have given examples of an appreciable effect of other factors, both heliospheric (asteroid and comet hazards) and galactic: galactic cosmic rays and 


\section{Years of Sunspot Observations}

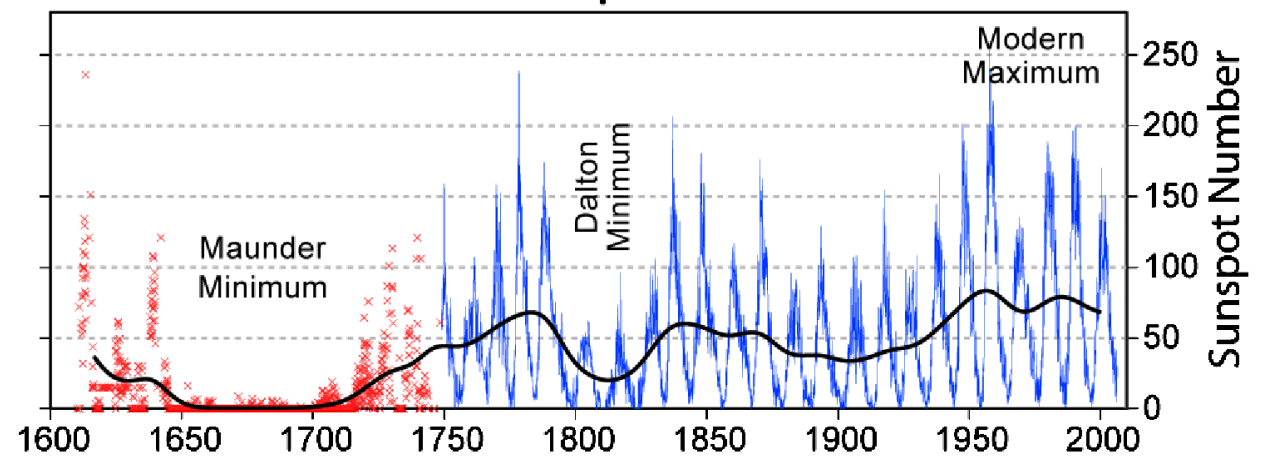

Figure 1. Solar activity since the invention of the telescope (1610): the degree of confidence of the observational data is shown graphically; red crosses: isolated observations; light-blue curve: restored monthly average Wolf numbers (low confidence); dark-blue curve: trustworthy monthly average Wolf numbers (http://commons.wikimedia.org/wiki/Image:Sunspot_Numbers.png).

ultrahard X-ray bursts from nonstationary galactic objects (the event of 27 December 2004).

[5] A vivid example of the asteroid and comet hazards was vividly demonstrated by the fall of fragments of Comet Shoemaker-Levy 9 on Jupiter in November 2001; it showed that such an event, though infrequent, is however quite real. This is confirmed by the discovery of giant craters (ring structures) on the Earth's surface; these craters were formed by impacts of rather large $(<1 \mathrm{~km})$ asteroids or fragments of comets. The comprehension of this risk has resulted in the creation of Near-Earth Asteroid Tracking Service, which follows significant Solar System objects passing in the immediate proximity from the Earth (asteroids, comets). The list of such potentially hazardous objects is regularly published on the Web page http://www.spaceweather.com/.

[6] Flows of galactic cosmic-ray particles arrive to the Earth from all directions of space. They consist of nuclei of chemical elements from hydrogen to uranium in proportions that approximately match the average element abundances in the Universe. At present the main presumed sources of galactic cosmic rays are supernova explosions. In the heliosphere at the Earth's orbit the energy spectrum of particles in these flows has a sharp peak at energies $0.5-1 \mathrm{GeV}$; the exact peak position depends on the state of the interplanetary magnetic field of the Solar System; in its turn, the latter is determined by the solar activity level. The flux of galactic cosmic ray particles changes in antiphase with solar activity variations [Panasyuk et al., 2006].

\section{Cyclicity of Solar Activity}

[7] It is accepted to call solar activity the entire set of phenomena in the solar atmosphere that change its radiation in various ranges of electromagnetic waves and various-energy particle fluxes. In the optical range these changes are exhibited basically in the change of the number of various solar structures; they are almost invisible in the optical radiation $(\sim 0.1 \%)$. However, in soft $\mathrm{X}$ rays these changes are ob- servable both in the number of structures and in radiation: the difference in the "background" radiation level in the 1$8 \AA$ range $(1-12.5 \mathrm{keV})$ at the solar activity maximum and minimum exceeds two orders of magnitude (more than 100 times). The solar activity state is characterized by some observational indices; of these, the longest time series exists for the relative sunspot number $(W)$ introduced for the first time by $\mathrm{R}$. Wolf. This index was determined on a regular basis at first in Zürich and since 1849 until now in Brussels. This year may be considered as the beginning of scientific observations of the Sun. In addition, Wolf restored monthly average values of the given index since 1749 and annual averages since 1700 using rather fragmentary data of individual European observers. Using literary data of occasional telescopic observations, modern explorers have managed to qualitatively prolong this series to 1611 . The reliability of all retrieved data is low; they enable us only to see a hint that the cyclic solar activity existed also at that time. One of the most remarkable features of the Sun are nearly-periodic changes of intensity or number of different manifestations of solar activity (SA)-solar activity cycles. For the first one, the cycle that began in March 1755 is conventionally adopted. Figure 1 shows the entire known series of relative sunspot numbers since 1610 until present; the confidence of the observational data is shown graphically; crosses: isolated observations (qualitative estimate), light-gray curve: low veracity; darker curve: reliable data. It should be noted that the analysis and comparison of Wolf number series for the periods 1749-2006 (numbered series) and 1849-2006 (reliable series) has shown obvious differences in the positions and magnitudes of the fundamental harmonics and spectral parameters of these series. In addition, the comparison of the series infers:

- a different behavior of the main characteristics on the interval 1749-1849;

- an increase in the length of the series results in a poorer resolution of some significant spectral characteristics (usually vice versa); 
Table 1. Solar activity cycles 1-23

\begin{tabular}{|c|c|c|c|c|c|c|c|c|}
\hline No. & $\begin{array}{c}\text { Beginning } \\
\text { of cycle }\end{array}$ & $\begin{array}{l}\text { Maximum } \\
\text { of cycle }\end{array}$ & $\begin{array}{l}\text { Minimum } \\
\text { of cycle }\end{array}$ & $W_{\min }^{*}$ & $W_{\max }^{*}$ & $\begin{array}{l}\text { Duration } \\
\text { of cycle }\end{array}$ & $\begin{array}{l}\text { Branch } \\
\text { of growth }\end{array}$ & $\begin{array}{l}\text { Branch } \\
\text { of decay }\end{array}$ \\
\hline 1 & 1755 III & $1761 \mathrm{VI}$ & $1766 \mathrm{~V}$ & 8.4 & 86.5 & 11.25 & 6.25 & 5.0 \\
\hline 2 & $1766 \mathrm{VI}$ & 1769 IX & $1775 \mathrm{~V}$ & 11.2 & 115.8 & 9.0 & 3.25 & 5.75 \\
\hline 3 & $1755 \mathrm{VI}$ & $1778 \mathrm{~V}$ & 1784 VIII & 7.2 & 158.5 & 9.25 & 2.92 & 6.33 \\
\hline 4 & 1784 IX & $1788 \mathrm{II}$ & 1798 IV & 9.5 & 141.2 & 13.67 & 3.42 & 10.25 \\
\hline 5 & $1798 \mathrm{~V}$ & $1805 \mathrm{II}$ & $1810 \mathrm{VII}$ & 3.2 & 49.2 & 12.25 & 6.75 & 5.5 \\
\hline 6 & 1810 VIII & 1816 IV & 1823 IV & 0 & 48.7 & 12.75 & 5.67 & 7.08 \\
\hline 7 & $1823 \mathrm{~V}$ & 1829 XI & $1833 \mathrm{X}$ & 0.1 & 71.7 & 10.50 & 6.50 & 4.0 \\
\hline 8 & 1833 IX & 1837 III & $1843 \mathrm{VI}$ & 7.3 & 146.9 & 9.67 & 3.33 & 6.33 \\
\hline 9 & $1843 \mathrm{VII}$ & $1848 \mathrm{II}$ & $1855 \mathrm{XI}$ & 10.5 & 131.6 & 12.42 & 4.58 & 7.83 \\
\hline 10 & $1855 \mathrm{XII}$ & 1860 II & 1867 II & 5.2 & 97.9 & 11.42 & 4.17 & 7.25 \\
\hline 11 & 1867 III & 1870 VIII & $1878 \mathrm{XI}$ & 2.2 & 140.5 & 11.92 & 3.42 & 8.50 \\
\hline 12 & $1878 \mathrm{XII}$ & 1883 XII & 1890 II & 5.0 & 74.6 & 11.33 & 5.0 & 6.33 \\
\hline 13 & 1890 III & 1894 I & 1901 XII & 2.6 & 87.9 & 12.08 & 3.83 & 8.25 \\
\hline 14 & 1902 I & 1906 II & 1913 VII & 1.5 & 64.2 & 11.67 & 4.08 & 7.59 \\
\hline 15 & 1913 VIII & 1917 VIII & 1923 VII & 5.6 & 105.4 & 10.08 & 4.0 & 6.08 \\
\hline 16 & 1923 VIII & $1928 \mathrm{IV}$ & 1933 VIII & 3.4 & 78.1 & 10.25 & 4.67 & 5.58 \\
\hline 17 & 1933 IX & 1937 IV & 1944 I & 7.7 & 119.2 & 10.5 & 3.58 & 6.92 \\
\hline 18 & 1944 II & $1947 \mathrm{~V}$ & 1954 III & 3.4 & 151.8 & 10.25 & 3.25 & 7.00 \\
\hline 19 & 1954 IV & 1958 III & 1964 IX & 9.6 & 201.3 & 10.42 & 3.92 & 6.50 \\
\hline 20 & $1964 X$ & $1968 \mathrm{XI}$ & $1976 \mathrm{VI}$ & 12.2 & 110.6 & 11.83 & 4.08 & 7.75 \\
\hline 21 & $1976 \mathrm{VI}$ & 1979 XII & 1986 VIII & 13.2 & 164.5 & 10.25 & 3.50 & 6.75 \\
\hline 22 & 1986 IX & 1989 VII & $1996 \mathrm{~V}$ & 8.0 & 158.11 & 9.58 & 2.92 & 6.66 \\
\hline 23 & $1996 \mathrm{VI}$ & 2000 IV & $2006 X I I$ & & 120.7 & 10.6 & 3.83 & 6.8 \\
\hline
\end{tabular}

$W^{*}$ : relative sunspot numbers smoothed over 13 months. Boldface: reliable SA cycles; italics: predicted values.

- an appreciable distortion of the "high frequency" part of the spectrum.

[8] All this puts in question the correctness of using the retrieved part of the series in the majority of applications. In other words, to study the effect of solar activity on various long-term processes, one can use only the reliable series of Wolf numbers (1849-2006), from the middle of cycle 9 to cycle 23 of solar activity. The small statistics of reliable solar cycles (14) and absence of a physical model of a solar cycle development puts so far insuperable barriers to the reliability of solar cycle prediction prior to its beginning. However, the situation changes with the beginning of a new cycle: after 18-24 months of its development, it is already possible to determine its height, the epoch of its maximum, and probable duration of the current solar cycle. For the last two cycles the most successful prediction of the solar cycle development its beginning was done by the Waldmeier method and by the method of solar cycle sets similarity proposed by the author [Ishkov, 2003, 2005]. Table 1 lists the characteristics of all "numbered" solar cycles.

[9] Almost eleven years of the development of the current cycle 23 (Figure 2) have completely uncovered its characteristics and the majority of features of its evolution. The current cycle is the second component of the physical 22-year solar cycle, and the greatest surprise was that, contrary to the Gnevyshev-Ol' rule, the trailing odd cycle 23, for the first time in the entire realistic (since 1849) history of the research of solar cycles, has become smaller than the leading even cycle 22. The main stages of the current solar cycle development [Ishkov, 2005] are as follows:

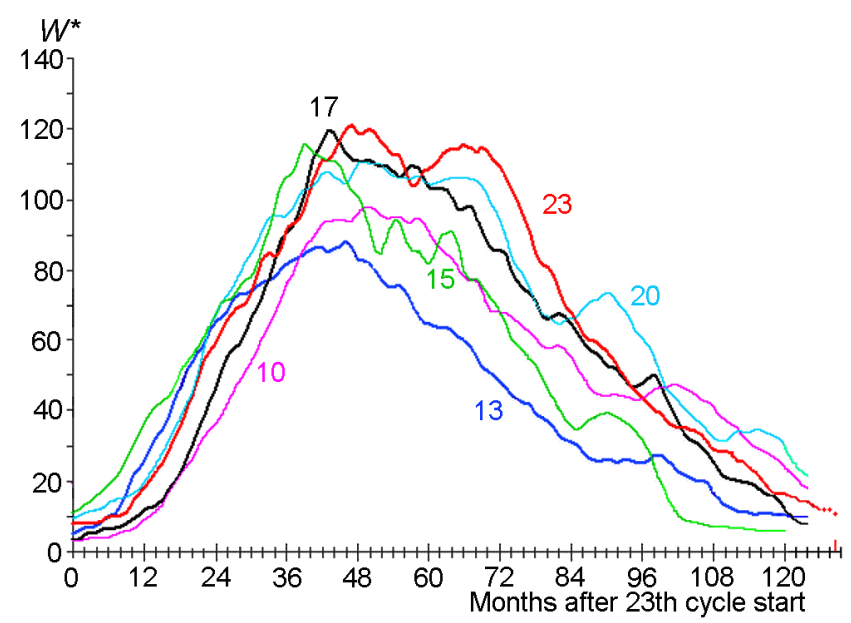

Figure 2. The development (131 months) of the current solar cycle 23 in comparison to similar mean-intensity solar cycles; $W^{*}$ : smoothed over 13 months of relative sunspot numbers (Wolf numbers). 
Table 2. Main parameters of flare events as functions of the intensity of the magnetic field in which they take place

\begin{tabular}{|c|c|c|c|c|c|c|c|c|}
\hline \multirow{2}{*}{$\begin{array}{l}\text { Magnetic } \\
\text { field }(\mathrm{G})\end{array}$} & \multirow{2}{*}{$\begin{array}{l}\text { Magnetic } \\
\text { class }\end{array}$} & \multirow{2}{*}{$\begin{array}{l}\text { Event } \\
\text { type }\end{array}$} & \multicolumn{2}{|c|}{ Maximum importance } & \multirow{2}{*}{$\begin{array}{c}F_{\mathrm{cm} \max } \\
\mathrm{sfu}\end{array}$} & \multirow{2}{*}{$\begin{array}{c}\mathrm{X}_{\text {hard }}, \\
\gamma\end{array}$} & \multirow[t]{2}{*}{ CME } & \multirow[t]{2}{*}{ FAS } \\
\hline & & & $\mathrm{H}_{\alpha}$ & $1-8 \AA$ & & & & \\
\hline$<50$ & - & SFE & brigtnenings & $\leq \mathrm{C} 7$ & $<50$ & - & + & + \\
\hline$<500$ & - & $\mathrm{SF}$ & $4 \mathrm{~N}$ & $\leq \mathrm{M} 7$ & $\leq 300$ & - & + & + \\
\hline$\leq 2000$ & $\gamma, \delta$ & $\mathrm{SF}$ & $3 \mathrm{~B}$ & $>\bar{X} 17.5$ & $>10000$ & + & + & + \\
\hline$>2500$ & $\delta$ & $\mathrm{SF}$ & $1 \mathrm{~B}$ & $\leq \mathrm{M} 5$ & $<10000$ & + & - & - \\
\hline
\end{tabular}

SFE: solar filament eruption; SF: solar flares; $F_{\text {cm max }}$ : maximum flux of centimeter-wavelength radio emission in solar flux units $(\mathrm{sfu}) ; \mathrm{X}_{\text {hard }}, \gamma$ : significant radiation in hard $\mathrm{X}$ rays and $\gamma$ rays; CME: coronal mass ejection; FAS: flare arch systems.

- $\operatorname{minimum}$ of the solar cycle 22: May $1996\left(W^{*}=8.0\right)$;

- beginning of the 23rd cycle phase of growth: September 1997;

- maximum of the relative sunspot number: April 2000 $\left(W^{*}=120.7\right)$

- global polarity reversal of the solar global magnetic field: July - December 2000;

- secondary maximum of the relative sunspot number: November $2001\left(W^{*}=115.8\right)$;

- maximum of the radio flux at a wavelength of $10.7 \mathrm{~cm}$ : February $2002\left(F^{*}=197 \mathrm{sfu}\right)$;

- phase of the current cycle maximum: October 1999 June 2002;

- beginning of the decay phase: July 2002;

- beginning of the minimum phase: July 2006.

- The most powerful flare events in the current cycle: October - November 2003.

\section{Geoeffective Solar Phenomena}

[10] The solar studies of the last decade have left no doubt that geoeffective phenomena on the Sun that completely determine the state of near-Earth space are extremely large flare events and coronal holes. The class of flare phenomena includes:

- solar flares with all the spectrum of dynamic manifestations of the motion of material and radiation in all ranges of the electromagnetic spectrum;

- solar filament eruptions with all accompanying phenomena.
[11] Solar flares take place in active regions with sunspot groups and without them; they represent a response of the solar atmosphere to a fast energy release, which results in a sharp local heating of all layers of the solar atmosphere accompanied by generation of powerful radiation in a broad band of the electromagnetic spectrum, from $\gamma$-ray quanta to kilometer radio waves as well as by flows of electrons, protons, and heavy nuclei. Solar filaments are clouds of dense and cooler (than the surrounding medium) plasma, which take in the magnetic field of the solar atmosphere the form of structures extended along the polarity reversal line. The mean sizes of solar filaments: length is 50 megameters (Mm), width is several megameters, and height above the visible surface of the Sun (photosphere) is $10 \mathrm{Mm}$. Solar filament eruptions observed with a high resolution have an appearance of a two-ribbon flare with a slowly growing intensity before the maximum ( $>1$ hour) and a long timescale of the intensity decline ( $>3$ hours). In the great majority of cases the phenomenon takes place outside active regions, in weak magnetic fields $(<50 \mathrm{G})$

[12] Flare phenomena (Figure 3) comprise the entire set of dynamic phenomena on the Sun; they are a consequence of the interaction of a new emerging magnetic flux with the already present magnetic fields. This definition includes all the spectrum of brightenings, flares, and solar filament eruptions, whose development follows the same scenario. The study [Ishkov, 1998] of the entire set of flare phenomena has made possible a classification of flare phenomena depending on the intensity of the magnetic field in which they take place (Table 2).

[13] In the great majority of cases, solar filament eruptions take place outside active regions, in weak magnetic fields (<50 G) (see Figure 4). "Spotless" flares are phenomena that take place in spotless active regions with a magnetic field of $<500 \mathrm{G}$. They are characterized by a slow growth toward the maximum and by very weak hard X-ray radiation; the microwave burst is always less than $300 \mathrm{sfu}$, and the X-ray importance is usually $\leq \mathrm{M} 7$; in the optical range their importance can reach the highest values, $4 \mathrm{~N}$. Long-lived flares in sunspot groups differ by maximum energy output in all ranges. Flares arising in the strongest magnetic fields $(>2000 \mathrm{G})$ are always impulsive ones; they 


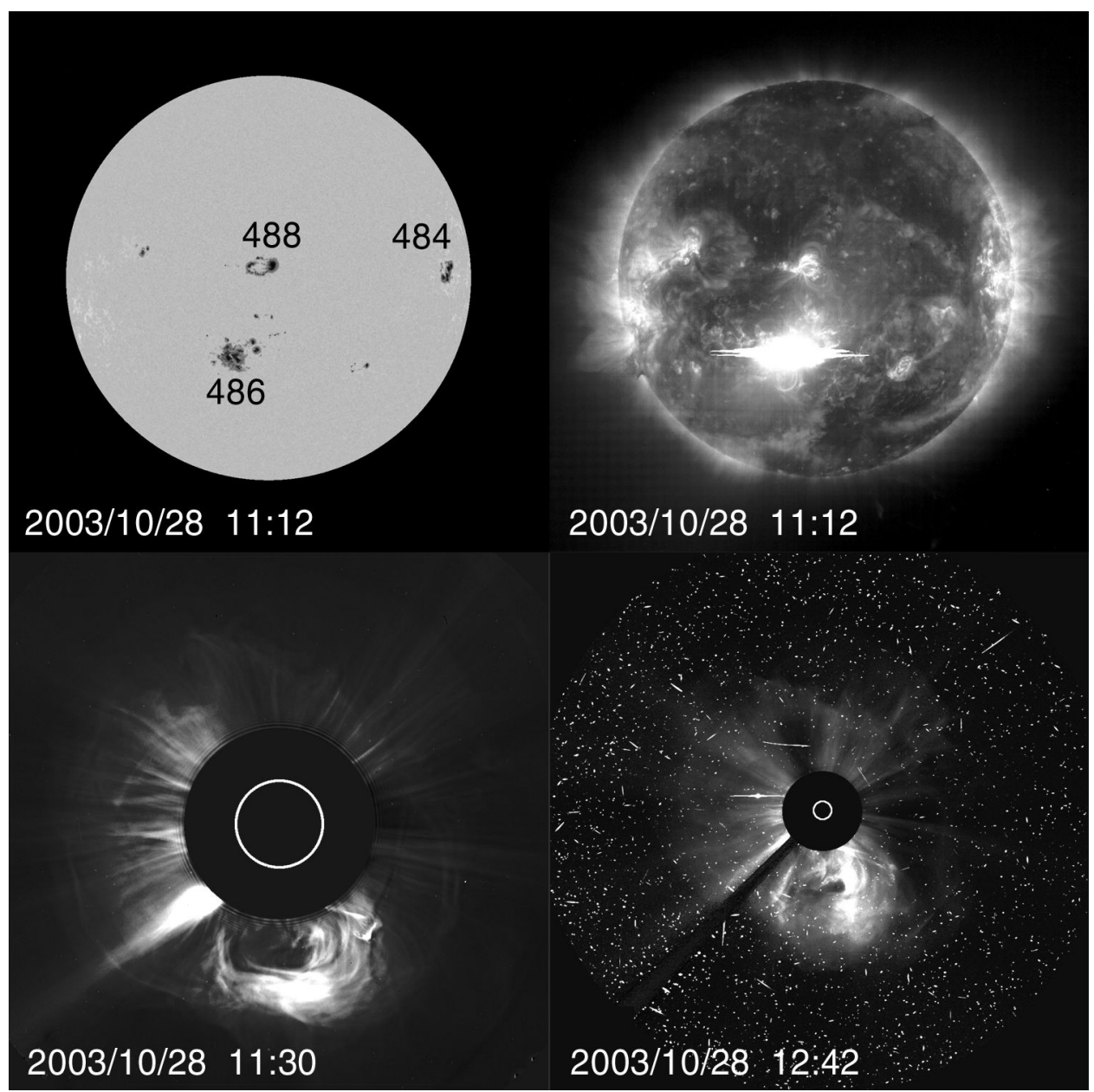

Figure 3. The solar situation during the extreme solar flare of 28 October 2003; upper row, left: the white-light Sun with sunspot groups (SOHO); upper row, right: the Sun in the extreme ultraviolet line $\lambda=195 \AA$ at the flare maximum (SOHO); lower row, left: coronal mass ejection from the flare (SOHO $\mathrm{C} 2$ coronagraph); lower row, right: the same coronal mass ejection at a greater distance from the Sun (SOHO C3 coronagraph).

are well visible in hard $\mathrm{X}$ rays and $\gamma$ rays up to the highest energies; however, their X-ray importance does not reach M5, and the optical importance does not exceed 1B.

[14] Using the observations of emerging magnetic fluxes (EMF), we can summarize phenomena after which the flare activity increases [Ishkov, 1998]:

- any appearance of a new EMF results in an flare activity increase;

- for the implementation of large solar flares it is necessary that the new EMF be rather large $\left(>10^{13} \mathrm{~Wb}\right)$ and the rate of its emergence be not less than $10^{9} \mathrm{~Wb} / \mathrm{s}$;

- large solar flares occur one-two days after the detection of EMF within an active region;

- large- and medium-importance flares in active regions are clustered in series, trains Obashev et al., 1973; Ishkov, 1998], which take place in a restricted time interval.
[15] The time interval in which the major part of largeand medium-importance flares is implemented in an active region is called period of flare energy release. Depending on the degree of development of an active region, characteristics of its magnetic field, and power of the new EMF, this period can take from 16 to 80 hours, on the average $55 \pm 30$ hours, or $16 \%$ of the transit time of an active region across the visible solar disk [Ishkov, 1998]. These tags allow us to predict the period of flare energy release one-two days before the occurrence of large geoeffective solar flares.

[16] The prediction of solar filament eruptions is more difficult: only direct observations of an EMF in weak magnetic fields can give a possibility to predict them. EMF interacts with the background magnetic field and can emerge only to regions of the neutral line of the axial magnetic-field component.

[17] Coronal holes are regions in the solar atmosphere with a magnetic field open to the interplanetary space; from them, solar plasma freely outflows to the heliosphere, forming high- 


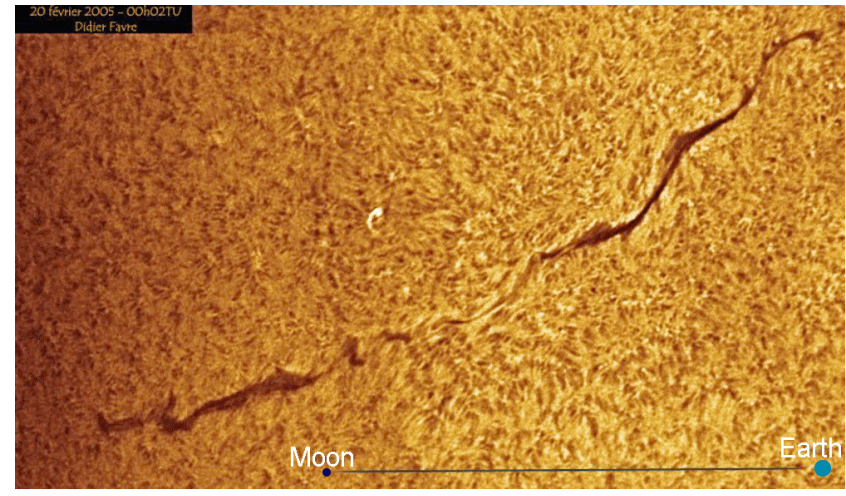

Figure 4. Filaments and prominences on the Sun in the extreme ultraviolet line $\lambda=304 \AA$ on 23 September 1999.

speed flows of the solar wind (see Figure 5). A large coronal hole usually exists during $4-8$ solar revolutions virtually without changing its position. However, its visible boundaries may displace by up to $20^{\circ} /$ day, changing its sizes or moving it as a whole. Low-latitude coronal holes are considered as sources of recurrent magnetic storms and of flows of energetic $(>2 \mathrm{MeV}$ ) electrons. Geoeffective coronal holes are those found in a heliolatitude interval of N25-S25, having a heliolatitude extension of $\geq 10^{\circ}$, area of $\geq 5000 \mathrm{msh}$ [Joselyn, 1986], and localized in enhanced background magnetic fields. The effect on the near-Earth space appears when the western boundary of the coronal hole reaches a heliolongitude of $\sim \mathrm{W} 40, \sim 3$ days after the transit of the western boundary of the coronal hole across the central meridian of the Sun [Watari, 1990; Solodyna et al., 1977]. We should especially emphasize the role of a coronal hole as an enhancer of the geoefficiency of solar flare phenomena. The presence of a coronal hole near active regions where a solar flare event takes place sharply increases their geoefficiency and expands the range of their localization. An example is the event of April 14, 1994, when an eruption of a high-latitude (S50) filament located beneath a large coronal hole resulted in a complete modification of magnetic structures in the southern hemisphere of the Sun [McAllister et al., 1995] and in a major magnetic storm of 17 April 1994.

[18] The electromagnetic disturbances from flare events appear virtually at the instant of the process development; corpuscular and plasma disturbances from solar geoeffective phenomena (flare events, coronal holes) propagating in the heliosphere, through the solar wind, affect the magnetospheres of planets, their satellites, and comets, causing considerable deviations from the background quiet state in nearly all layers of the considered objects. The agents causing this disturbance are:

- transient structures, coronal mass ejections, which are a consequence of active processes in flares and filament eruptions; their direct observations enable us to specify the direction of a disturbance motion in the interplanetary space and the possibility of its incoming to near-Earth space;

- high-velocity flows of solar plasma following a shock wave from powerful flare events or outflowing from regions with an opened magnetic configuration (coronal holes).

\section{Effect on Near-Earth Space (Space Weather)}

[19] The full chain of disturbances from in an individual large flare event can be represented in three separate stages of the effect (http://sec.noaa.gov/NOAAscales):

[20] Electromagnetic shock reaches near-Earth space at the speed of light, i.e., at the instant of the development of a solar flare. During its effect in short-wave ranges of electromagnetic radiation the flux increases by several orders of magnitude relative to the background values. For instance, in soft X rays (1-8 $\AA, 12.5-1 \mathrm{keV})$ the flux increase can reach four orders. As a consequence, immediately at the instant of of a flare development (timescale of the disturbance development is a few minutes after the beginning of the flare) sudden ionospheric disturbances develop in nearEarth space, causing a complete radio silence in the range of high frequencies at the dayside of the Earth; its duration reaches several hours, upsetting the radio communication with ships and airplanes. In the range of low frequencies at the dayside of the planet, errors in positioning systems of ground-based objects and satellites sharply increase and are accumulated during many hours. At present, a five-grade scale for the assessment of this kind of disturbances has been introduced: from R1 for flares with an X-ray importance of M (up to 950 events per cycle) to R5 for the most powerful flares with an X-ray importance of $\geq$ X17.5 (1-6 times per cycle).

[21] Ejection of energetic solar particles (protons, electrons, neutrons), which, reaching NES, cause solar proton events (SPE). Invasions of high-energy solar particles $\left(E_{p r}>10 \AA\right)$ sharply raise the level of radiation hazard to astronauts, crews and passengers of high-altitude airplanes at high latitudes, result in losses of satellites and in failures of scientific and navigation instruments on space objects, interrupt short-wave communication in subauroral regions, and provoke a sharp increase in positioning system errors. This results in serious problems in positioning of ground-based and space objects. The timescale of the SPE onset with
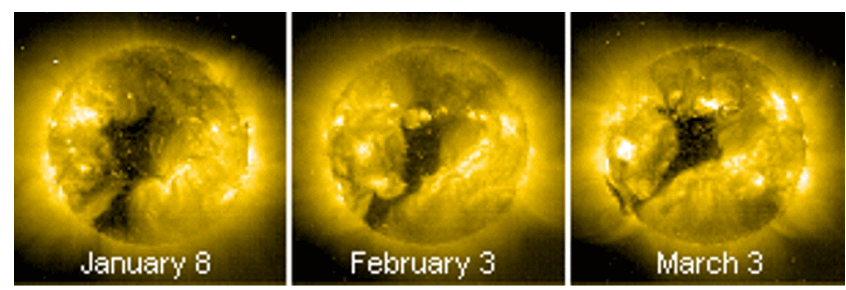

Figure 5. A coronal hole at three 2002 solar revolutions in the extreme ultraviolet line $\lambda=284 \AA$ (SOHO) 
respect to the beginning of a proton flare is a few hours, though particles with $\mathrm{GeV}$ energies arrive to the Earth at the speed of light. To describe SPE in near-Earth space, a five-grade scale of the protons flux assessment with energies $E>10 \mathrm{MeV}$ has also been introduced: from $S 1$ for a proton flux of 10 particles per second per steradian per square centimeter (up to 50 events per cycle) to $S 5$ for a proton flux by four orders of magnitude greater (less than one event per cycle).

[22] Plasma shock: interplanetary shocks and flows of solar plasma with enhanced density and/or speed (transient structures, high-speed solar wind flows) cause magnetic and ionospheric disturbances in near-Earth space. For disturbances of the geomagnetic field of the Earth with the intensity exceeding the threshold of magnetic storms, a fivegrade system of their estimation has also been introduced: from G1 for disturbances in which at least one three-hour $K p$ index has reached 5 ( $~ 900$ days per solar cycle) to G5 with $K p=9$ (1-5 days per cycle). It should be kept in mind that this scale estimates the intensity of a geomagnetic disturbance; it is quite possible to imagine a situation when the disturbance has an importance G1 or G2, but in fact there is no magnetic storm, and the diurnal geomagnetic index $A p$ is much lower than the magnetic storm threshold. Certainly, it is a problem of definition of "MAGNETIC STORM"magnetic disturbance with a duration not shorter than 12 hours and with a mean $A p$-index not lower than 27 (in western countries the threshold value is $A p=30$ ).

[23] At present the problem of reflection of the situation in near-Earth space and estimation of its state is solved by "space weather" services, which have been created in leading institutes and organizations all over the world (more than 40 URLs); the main ones are:

- USA (NOAA SEC, http://www.swpc.noaa.gov);

- Europe (ESA, http://www.esa-spaceweather.net/spweather/current_sw/index.html);

- World Data Center, solar activity indices, Brussels, http://sidc.oma.be/html/LatestSWData.html.) SUN);

- Japan (http://hiraiso.nict.go.jp);

- Australia (http://www.ips.gov.au/);

- Russia: Space Research Institute, Russian Academy of Sciences, http://www.iki.rssi.ru/sw.htm;

- Institute of a terrestrial magnetism, ionosphere and radiowave propagation, Russian Academy of Sciences, http://www.izmiran.ru.

[24] Their task is to give in real time the main characteristics of phenomena determining the NES state and the indices describing the state of the magnetosphere, ionosphere, and other layers of the Earth's atmosphere. The most informative Web pages of the space weather state are granted by the Space Weather Prediction Center of the National Oceanic and Atmospheric Administration (SWPC, NOAA, USA) http://www.swpc.noaa.gov/.

\section{Prediction of Geoeffective Solar Phenomena and the State of Near-Earth Space}

[25] Here we mean for the prediction of geoeffective solar phenomena a combination of all kinds of forecasts aiming to the calculation of the development of processes and phenomena taking place in the solar atmosphere and directly influencing the magnetic and radiation conditions in near-Earth space in the given time intervals. Time intervals for which the forecast is possible are determined by the characteristics of physical processes of emergence of new magnetic fluxes in the solar atmosphere, the character of their interaction with the already present magnetic field as well as regularities in the appearance and evolution of solar structures, such as solar flare events and coronal holes. The prediction of disturbances in near-Earth space directly depends on a successful, reliable forecast of solar geoeffective phenomena, such as large flare events and coronal holes. The observational data for the determination of the evolutionary and flare state of the Sun are stored at http://www.swpc.noaa.gov/Data/.

1. Daily characteristics of solar activity: solar activity indices $W, F_{10 \mathrm{~cm}}, X_{\mathrm{mp}}$; these data allow us to determine the phase of the current solar cycle and to estimate indirectly the flare activity level.

2. Heliographic coordinates of all sunspot groups; the shape, area, extension, and their evolutionary characteristics. These data yield the localization, dynamics of development, and information on the appearance of new EMF in individual active regions; together with the data about the previous revolution, this enables us to predict their flare activity for the subsequent period.

3. Daily data about solar flare events: heliographic coordinates of all significant flares, their localization and time characteristics, optical and X-ray importance, total flux of the burst in soft $\mathrm{X}$ rays, parameters of the radio bursts, and the presence of an ejection in the flare, coordinates, time, and sizes of solar filament eruptions. The localization and basic parameters of flare events allow us to implement diagnostics of the geoefficiency of a flare and to estimate the possibility of arrival of a disturbance and of solar particles to the Earth.

4. Information on coronal mass ejections accompanying flare events allows us to specify the possibility of arrival of a disturbance and of solar particles to the Earth.

5. Information about low-latitude coronal holes: their localization, area, sign of the magnetic field in which it was formed, dynamics and position of the IMF sector. This information enables us to trace the phenomena determining the successful prediction of the recurrent geomagnetic activity. 
[26] The observational data on the interplanetary medium are presented on the Web page http://www.swpc.noaa.gov/ Data/.

1. Data from the ACE satellite on the solar wind and interplanetary magnetic field with a 1-min and 5-min time resolution, with 2-, 6-, and 24-h samples and with a 1-h time resolution for 3- and 7-day samples (http://www.swpc.noaa.gov/ace/).

2. Proton fluxes at the geostationary orbit with a 5-min resolution, GOES spacecraft (http://www.swpc.noaa. gov/).

3. Electron fluxes at the geostationary orbit with a 5-min resolution, GOES spacecraft (http://www.swpc.noaa. gov/).

[27] Indices and observational data on the geomagnetic field (http://www.swpc.noaa.gov/):

1. $A$ - and $K$-indices of all geomagnetic observatories for 30 days.

2. $A$ - and $K$-indices in real time from Fredericksburg and College Geomagnetic Observatories as well as the preliminary planetary $A p$-index for the Western Hemisphere.

3. Data of the magnetometer on the GOES geostationary satellite with a 1-min time resolution.

4. Hour and diurnal reviews(views) of the magnetometric data.

5. Data from the POES satellite on energetic particles of the Earth's radiation belts. The generalized picture of space weather in real time is presented on the page http://www.swpc.noaa.gov/today.html.

[28] A brief description for all interested in space weather is given on the Web page http://www.spaceweather.com, on which short articles on phenomena in NES, conditions of the observations of planets, comets, and meteor showers are published. The information on near-Earth asteroids and many other interesting articles on aurora, atmospheric phenomena, etc. are also published. The prediction of solar flare events on the basis of the analysis of emerging magnetic fluxes in active regions is implemented only in the Institute of Terrestrial Magnetism, Ionosphere, and Radiowave Propagation, Russian Academy of Sciences, since 1990. It is accessible to public within the framework of the weekly review of the state of near-Earth space, which appears on Mondays since 1997 at http://www.izmiran.rssi.ru/space/solar/forecast (Russian version) and http://titan.wdcb.ru/virbo_rus/viewlast.do? Section $=$ RBBulletin (English version). In the case of appearance in an active region of a new magnetic flux with a magnitude and ascent rate sufficient for the implementation of large flares, the Web page gives an appendix, in which the probable flare potential of the given active region is estimated.

[29] The situation on the Sun in a daily mode and attempts of warning about flare events are given on the following Web pages: of the Big Bear Observatory, USA:
- Big Bear Observatory, USA (http://www.bbso.njit. edu/cgi-bin/ActivityReport);

- Lockheed Martin Advanced Technology Center, Solar and Astrophysics Laboratory (http://www.lmsal.com /forecast/);

- System of observation and data processing Solar Soft (http://www.solarmonitor.org/index.php);

- NASA Goddard Space Flight Center's Solar Data Analysis Center (http://umbra.nascom.nasa.gov/).

[30] The prediction of space weather (geomagnetic field) invoking mainly solar-wind parameters from the data of the ACE (Advanced Composition Explorer) spacecraft located at the L1 libration point (this enables to forecast geomagnetic disturbances approximately $40 \mathrm{~min}$ prior to their beginning). The main ones are Space Weather Prediction Center of the National Oceanic and Atmospheric Administration (SWPC, NOAA, USA) (http://www.swpc.noaa.gov/ alerts/index.html) and Naval Research Laboratory (USA) (http://ppdweb.nrl.navy.mil/whatsnew/prediction/).

\section{Conclusion}

[31] From what was said we can conclude that prediction of the state of near-Earth space is now possible and is sufficiently exact. At the first stage, one-two days beforehand, using observations of emerging new magnetic fluxes, we predict the time interval in which, with a probability not less than $90 \%$, large flare events will take place; their probable geoefficiency in all three space weather positions is estimated. At the second stage, during or immediately after a flare event, the prediction is updated using electromagnetic radiation as well as CME and

(1) the probability of the arrival of solar high-energy particles (solar proton event) to near-Earth space, their probable maximum fluxes and duration are given (prognostic interval from 1 to 6 hours, prediction probability is about $70 \%$ );

(2) the probability of a geomagnetic disturbance, its probable intensity, and duration as well as parameters of a probable ionospheric disturbance and aurora are assessed (prognostic interval from 17 hours to 5 days, probability of the forecast is not lower than $70 \%$ ).

[32] The third stage consists in refinement of the prediction of the geomagnetic disturbance intensity and duration involving the parameters of structures in the perturbed solar wind using SOHO data (stationary orbit at 1.5 million kilometers from the Earth). Approximately $40 \mathrm{~min}$ before its beginning, it is concluded whether the geomagnetic disturbance will reach the level of a magnetic storm; its magnitude, intensity, and duration are predicted (prediction probability is not lower than $80 \%$ ).

[33] At present the technique of prediction of large geoeffective solar flare events from observations of emerging new magnetic fluxes has passed a successful check by the operation of the Russian research satellites GRANAT, GAMMA, 
CORONAS-I, and CORONAS-F; this technique is successfully applied to realistic practical tasks.

[34] Acknowledgments. The author thanks the SOHO MDI EIT, and LASCO teams for the opportunity to use the data of their observations for the illustration of active solar events. This work was supported by the Russian Foundation for Basic Research (project codes 07-02-13525 and 07-02-00246) as well as by Program No. 16 of the Presidium of the Russian Academy of Sciences.

\section{References}

Ishkov, V. N. (1998), Emerging magnetic fluxes: a key to prediction of large solar flares, Izv. RAN. Ser. fiz., 62(9), 1835.

Ishkov, V. N. (2003), The current 23 cycle of solar activity: its evolution and principal features, in Solar variability as an input to the Earth's environment. International Solar Cycle Studies (ISCS) Symposium, p. 103, ESA Publications Division, ESA SP-535, Noordwijk.
Ishkov, V. N. (2005), Properties of the current 23-rd solar activity cycle, Solar System Res., 39(6), 1, doi:10.1007/s11208005-0058-6.

Joselyn, (1986), SESC methods for short-term geomagnetic prediction, in Solar-Terrestrial Predictions: Proceedings of a Workshop at Meudon, France, June 18-22, 1984, p. 404, Edited by P. A. Simon, G. Heckman, and M. A. Shea, NOAA, Boulder, CO.

McAllister, A. H., M. Dryer, P. McIntosh, H. Singer, and L. Weiss (1995), A quiet CME and a severe geomagnetic storm: April 14-17, 1994, Proc. of the 2nd SOLTIP Symposium, GBRSC News, no. 5, 191.

Obashev, S. O., et al. (1973), Solar activity, in Tr. Astrofiz. Inst. (in Russian), vol. 23, p. 103, Nauka, Alma-Ata.

Panasyuk, M. I., et al. (2006), Radiation conditions in space (in Russian), 132 pp., Biblion - Russian Book, Moscow.

Solodyna, C. V., A. S. Krieger, and J. T. Nolte (1977), Observations of the birth of a small coronal hole, Solar Phys., 54(1), 123, doi:10.1007/BF00146428.

Watari, S. I. (1990), The latitudinal distribution of coronal holes and geomagnetic storms, in Proceeding of the Solar-Terrestrial Predictions Workshop, vol. 1, p. 627, NSO, Leura, Australia.

V. N. Ishkov, Institute of Terrestrial Magnetism, Ionosphere, and Radiowave Propagation, Russian Academy of Sciences, Troitsk, 142090 Russia (ishkov@izmiran.ru) 